\title{
Commentary: On helicon thrusters: Will they ever fly?
}

\author{
Kazunori Takahashi ${ }^{1 *}$, Christine Charles ${ }^{2}$, Rod W. Boswell ${ }^{2}$, Yoshinori Takao ${ }^{3}$, \\ Amnon Fruchtman ${ }^{4}$, Jaume Navarro-Cavallé ${ }^{5}$ and Mario Merino ${ }^{5}$ \\ ${ }^{1}$ Department of Electrical Engineering, Tohoku University, Sendai, Japan, ${ }^{2}$ Space Plasma, Power and Propulsion Laboratory, \\ Research School of Physics, The Australian National University, Canberra, ACT, Australia, ${ }^{3}$ Division of Systems Research, \\ Yokohama National University, Yokohama, Japan, ${ }^{4}$ Faculty of Sciences, Holon Institute of Technology (HIT), Holon, Israel, \\ ${ }^{5}$ Equipo de Propulsión Espacial y Plasmas (EP2), Universidad Carlos III de Madrid, Leganés, Spain
}

Keywords: helicon thruster, electric propulsion, radiofrequency plasma, magnetic nozzle, magnetic field

\section{A Commentary on}

On helicon thrusters: Will they ever fly?

by Godyak, V. (2020). J. Appl. Phys. 127:103301. doi: 10.1063/1.5139998

\section{OPEN ACCESS}

Edited by:

Earl E. Scime,

West Virginia University, United States

Reviewed by:

Mitchell L. R. Walker,

Georgia Institute of Technology,

United States

*Correspondence:

Kazunori Takahashi

kazunori@ecei.tohoku.ac.jp

Specialty section:

This article was submitted to

Plasma Physics,

a section of the journal

Frontiers in Physics

Received: 07 May 2020

Accepted: 22 June 2020

Published: 20 August 2020

Citation:

Takahashi K, Charles C, Boswell RW,

Takao Y, Fruchtman A,

Navarro-Cavallé $J$ and Merino $M$

(2020) Commentary: On helicon

thrusters: Will they ever fly?

Front. Phys. 8:277.

doi: 10.3389/fphy.2020.00277
A recent paper [1] has discussed and questioned the suitability of the helicon thruster (HT) for space use, concluding that "It seems unlikely that a spacecraft based on an HP thruster will ever fly (Sic)," by comparing the Helicon Plasma (HP) source with an inductively coupled plasma (ICP) source, in terms of radiofrequency (RF) power coupling, controllability, and disadvantage of magnetic field requirement. [1] presents no original data and instead uses a narrow set of data selectively drawn from the literature to derive inaccurate and biased comments such that appear in the abstract "It is shown that helicon thrusters should be inferior to those based on inductively coupled plasmas (Sic)" and "we have shown that several key features of the HP thrusters make them inferior to thrusters based on ICP or FMICP (Sic)."

The main discussion in [1] focuses on the plasma production, the presence of capacitive coupling and its effect on the power coupling. There is, however, no scientific discussion on thrust generation and thruster performance yielding a disconnect between the manuscript's content and its title, abstract, and conclusion: as a clear example, Sec. VI in [1] concludes that the magnetic field does not provide any advantage to the HT despite citing $[2,3]$, which provide evidence of thrust generation by applying the magnetic field. Although power coupling is one of many important issues in improving any electric thruster performance, the thrust is given by the momentum flux (i.e., density and velocity) exhausted from the propulsion system. Godyak [1] entirely neglects the processes involved in the exhaust of the plasma from the source, the momentum conversion/loss, and the energy loss in the source: this commentary addresses these processes and how they can be affected by the magnetic field.

1) B-field effect on the HT performance: Previous thrust measurement has shown an increase in the thrust of the HT when applying a magnetic field while maintaining a constant plasma density in the source [4]. For constant plasma density in the source, the thrust increases with an increase in the magnetic field. For a RF power of $\sim 1 \mathrm{~kW}$, the thrust with no magnetic field is $\sim 3-4 \mathrm{mN}$, whereas the total thrust is larger than $10 \mathrm{mN}$ for a magnetic field strength of $\sim 700 \mathrm{G}$ corresponding to an electric power of less than $1 \mathrm{~kW}$ consumed by the solenoid. The thrust increase is caused by momentum conversion which results from the Lorentz force in the magnetic nozzle, as previously investigated in analytical [5], numerical [6, 7], and experimental studies [8-10]. Therefore, applying the magnetic field is more effective than increasing the RF power. Although the magnetic field sometimes degrades the RF power coupling as discussed from Figure 4 in [1], the thruster performance would be the result of the balance between the advantage of applying a magnetic field 
and the plasma production efficiency. Furthermore, a high plasma potential in the source can be obtained by applying the magnetic nozzle, resulting in a high exhaust velocity of the ions and an increase in specific impulse [11, 12].

2) B-field effect on the loss in the HP source: [1] claims that the effect of the magnetic field on the energy balance owing to the radial confinement is little for such an open system (Sec. V). However, a recent experiment providing the energy and momentum fluxes lost to the radial wall has clearly shown that the energy and momentum losses can be inhibited by the magnetic field [13]. In electric propulsion devices, it is important to increase the plasma flux exhausted from the source. An experiment performed by Little and Choueiri [14] has clearly shown the significant role of the magnetic field on the plasma exhaust from the source exit.

3) Power absorption profile: [1] claims in Sec. V that "the plasma density, its spatial profile, and electron temperature are the same for MWP, ICP, and HP (Sic)." However, a fundamental experiment performed by Lafleur et al. [15] has clearly shown modification of the plasma density profile owing to the presence of the Helicon wave. Moreover, HT performance depends not only on the density profile but also on the electron temperature and the RF power deposition maps. A numerical model has shown that the latter is controlled by the magnetic field [16]. The effect of the density profile on the thrust has been experimentally and analytically demonstrated by combining thrust measurements and plasma parameters profile measurements $[6,17,18]$.

4) Power requirement for the magnetic field: The power consumed by the solenoid is an important issue as pointed out in [1], of course, for designing a HT system. For high RF power operation of the thruster, the fraction of electricity required for

\section{REFERENCES}

1. Godyak V. On helicon thrusters: will they ever fly? J Appl Phys. (2020) 127:103301. doi: 10.1063/1.5139998

2. Ahedo E. Plasma for space propulsion. Plasma Phys Control Fusion. (2011) 53:124037. doi: 10.1088/0741-3335/53/12/124037

3. Takahashi K. Helicon-type radiofrequency plasma thrusters and magnetic plasma nozzles. Rev Mod Plasma Phys. (2019) 3:3. doi: 10.1007/s41614-019-0024-2

4. Takahashi K, Charles C, Boswell RW. Approaching the theoretical limit of diamagnetic-induced momentum in a rapidly diverging magnetic nozzle. Phys Rev Lett. (2013) 110:195003. doi: 10.1103/PhysRevLett.110.195003

5. Fruchtman A. Electric field in a double layer and the imparted momentum. Phys Rev Lett. (2006) 96:065002. doi: 10.1103/PhysRevLett.96.065002

6. Ahedo E, Merino M. Two-dimensional supersonic plasma acceleration in a magnetic nozzle. Phys Plasmas. (2010) 17:073501. doi: 10.1063/1.3442736

7. Merino M, Ahedo E. Magnetic Nozzles for Space Plasma Thrusters. Encyclopedia of Plasma Technology. Boca Raton, FL: Taylor and Francis (2016). doi: 10.1081/E-EPLT-120053936

8. Takahashi $\mathrm{K}$, Lafleur $\mathrm{T}$, Charles $\mathrm{C}$, Alexander $\mathrm{P}$, Boswell RW. Electron diamagnetic effect on axial force in an expanding plasma: experiments and theory. Phys Rev Lett. (2011) 107:235001. doi: 10.1103/PhysRevLett.107.235001

9. Charles C, Takahashi K, Boswell RW. Axial force imparted by a conical radiofrequency magneto-plasma thruster. Appl Phys Lett. (2012) 100:113504. doi: $10.1063 / 1.3694281$ the solenoid would become small, e.g., less than $20 \%$ for $5 \mathrm{~kW}$ $\mathrm{RF}$ power and $1 \mathrm{kG}$ magnetic field requiring $\sim 900 \mathrm{~W}$ of power for Figure 22 in [3]. Furthermore, solenoids might be replaced by permanent magnets, as previously demonstrated $[19,20]$; this requires additional design optimization to produce the desired magnetic field.

Based on points 1 to 4 and current literature status, the HT operating with a magnetic field has shown better performance when compared with the ICP: Takahashi [3] reports a thruster efficiency increased to about $20 \%$ when using an applied magnetic field, which is higher than the typical ICP thruster [21]. Because the HT research and development is in its early stages with respect to other mature electric propulsion technologies (gridded ion and Hall effect thrusters), it is premature to conclude that the HT "should be inferior (Sic)" to the ICP thruster only from the viewpoint of power coupling as in [1]. The suitability criteria also include issues such as the allowed development timeframe and cost, the system's expected lifetime, and potential competitive advantages if successfully developed. There is on-going development work on RF thrusters of different types including the HT and the ICP, which aims at increasing performance and design based on space mission requirements; this includes improved designs of RF antenna, matching network, and RF power control, as shown in recent studies [22-24] including Godyak [1].

\section{AUTHOR CONTRIBUTIONS}

$\mathrm{KT}$, CC, and RB initiated the discussion on the commentary and wrote the first manuscript. The consideration described in the manuscript was discussed with YT, AF, JN-C, and MM. The manuscript was reviewed and revised by all the authors.
10. Correyero S, Merino M, Elias P-Q, Jarrige J, Packan D, Ahedo E. Characterization of diamagnetism inside an ECR thruster with a diamagnetic loop. Phys Plasmas. (2019) 26:053511. doi: 10.1063/1.5093980

11. Charles C. High source potential upstream of a current-free electric double layer. Phys Plasmas. (2005) 12:044508. doi: 10.1063/1.1883182

12. Takahashi K, Shida Y, Fujiwara T, Oguni K. Supersonic ion beam driven by permanent-magnets-induced double layer in an expanding plasma. IEEE Trans Plasma Sci. (2009) 37:1532-6. doi: 10.1109/TPS.2009.2024342

13. Takahashi K, Sugawara T, Akira A. Spatially- and vector-resolved momentum flux lost to a wall in a magnetic nozzle rf plasma thruster. Sci Rep. (2020) 10:1061. doi: 10.1038/s41598-020-58022-6

14. Little JM, Choueiri EY. Critical condition for plasma confinement in the source of a magnetic nozzle flow. IEEE Trans Plasma Sci. (2015) 43:277-86. doi: 10.1109/TPS.2014.2322522

15. Lafleur T, Charles C, Boswell RW. Plasma control by modification of helicon wave propagation in low magnetic fields. Phys Plasmas. (2010) 17:073508. doi: $10.1063 / 1.3460351$

16. Tian B, Merino M, Ahedo E. Two-dimensional plasma-wave interaction in an helicon plasma thruster with magnetic nozzle. Plasma Sources Sci Technol. (2018) 27:114003. doi: 10.1088/1361-6595/aaec32

17. Takahashi K, Takao Y, Ando A. Modifications of plasma density profile and thrust by neutral injection in a helicon plasma thruster. Appl Phys Lett. (2016) 109:194101. doi: 10.1063/1.4967193

18. Takahashi K, Charles C, Boswell RW, Ando A. Demonstrating a new technology for space debris removal using a bi-directional plasma thruster. Sci Rep. (2018) 8:14417. doi: 10.1038/s41598-018-32697-4 
19. Chen FF. Performance of a permanent-magnet helicon source at 27 and 13 MHz. Phys Plasmas. (2012) 19:093509. doi: 10.1063/1.4754580

20. Takahashi K, Charles C, Boswell R, Ando A. Performance improvement of a permanent magnet helicon plasma thruster. J Phys D: Appl Phys. (2013) 46:352001. doi: 10.1088/0022-3727/46/35/352001

21. Lafleur T, Takahashi K, Charles C, Boswell RW. Direct thrust measurement and modelling of a radio-frequency expanding plasma thruster. Phys Plasmas. (2011) 18:080701. doi: 10.1063/1.3610570

22. Charles C, Liang W, Raymond L, Rivas-Davila J, Boswell RW. Vacuum testing of a miniaturized switch mode amplifier powering an electrothermal plasma micro-thruster. Front Phys. (2017) 5:36. doi: 10.3389/fphy.2017. 00036

23. Liang W, Charles C, Raymond L, Stuchbery A, Surakitbovorn K, Gu L, et al. An integrated RF power delivery and plasma micro-thruster system for nano-satellites. Front Phys. (2018) 6:115. doi: 10.3389/fphy.2018.00115
24. Takahashi K, Hanaoka K, Ando A. Fast and automatic control of a frequency-tuned radiofrequency plasma source. Front Phys. (2020) 7:227. doi: 10.3389/fphy.2019.00227

Conflict of Interest: The authors declare that the research was conducted in the absence of any commercial or financial relationships that could be construed as a potential conflict of interest.

Copyright (C) 2020 Takahashi, Charles, Boswell, Takao, Fruchtman, Navarro-Cavallé and Merino. This is an open-access article distributed under the terms of the Creative Commons Attribution License (CC BY). The use, distribution or reproduction in other forums is permitted, provided the original author(s) and the copyright owner(s) are credited and that the original publication in this journal is cited, in accordance with accepted academic practice. No use, distribution or reproduction is permitted which does not comply with these terms. 